\title{
Luego de tres papanicolaou consecutivos normales podria extenderse el intervalo a tres años
}

Risk of Cervical Cancer Associated with Extending the Interval between Cervical Cancer Screenings. NEJM, October 16, 2003, 349:1501-1509

\section{¿Por qué este estudio?}

En Argentina, la proporción de mujeres con controles ginecológicos adecuados es muy baja. Según los datos más optimistas alcanza el $20 \%$ con grandes diferencias entre las distintas regiones. Por el contrario, la población que sí realiza rastreo de cáncer de cuello uterino (CCU) con papanicolaou (PAP) suele estar "sobre rastreada" con estudios anuales e incluso semestrales y habitualmente acompañados de colposcopía, cuando ya existe evidencia de que esta estrategia no solo no es costo-efectiva sino que además puede agregar comorbilidad. El intervalo entre la toma de PAP había sido definido en base a las características de la prueba diagnóstica, a la biología de la infección por el virus del papiloma humano (HPV) y a la progresión de la displasia a CCU. Sin embargo nunca había sido calculado el exceso de riesgo de extender este intervalo, lo que constituye el objetivo principal de este estudio.

A partir de este trabajo, la USPSTF (EEUU) recomienda desde enero de $2003^{1}$ rastrear con PAP a todas las mujeres mayores de 21 años o luego del inicio de las relaciones sexuales al menos cada tres años luego de tres PAP consecutivos con resultado normal, y hasta los 65 años. De esta manera se unifican con las recomendaciones de los canadienses ${ }^{2}$ y europeos ${ }^{3}$ quienes hacía tiempo se habían expedido en ese sentido.

\section{El estudio}

El objetivo principal de este trabajo fue determinar el exceso de riesgo de CCU asociado a la extensión del intervalo inter PAP a tres años en mujeres con tres citologías previas consecutivas negativas. Un objetivo secundario fue determinar el número de PAP y colposcopías necesarias para diagnosticar un caso de CCU con los distintos intervalos de rastreo.

Se basaron para esto en los datos del Programa del Centro de Control de Enfermedades (CDC) para la detección precoz del CCU que se lleva a cabo en EEUU para el rastreo de mujeres de bajos recursos y sin acceso al seguro social. Tomaron los resultados de los PAP realizados entre enero de 1991 y marzo de 2000. La mayoría había sido realizado mediante citología convencional y reportado según la clasificación de Bethesda. Excluyeron los PAP insatisfactorios, los pendientes, los no clasificados y aquellos en los que no se contaba con los datos de las pacientes.

Agruparon a las mujeres en cuatro categorías de rastreo: 1) un PAP obtenido por el programa del CDC; 2 ) un PAP negativo + otro PAP 3) dos PAP negativos + otro PAP; 4) tres PAP negativos + otro PAP. Se define como PAP negativo a aquel normal o con cambios celulares reactivos. Se define como pruebas consecutivas a aquellas con un intervalo menor a 36 meses entre sí. Fueron excluidos los PAP reportados con menos de nueve meses de intervalo ya que no se consideraron de rastreo, sino de seguimiento de patología.

Utilizaron el modelo matemático de Markov* para estimar el riesgo de cáncer basándose en la historia natural de la progresión del CCU dada la prevalencia de displasia y las características operativas (sensibilidad y especificidad) de la prueba de rastreo (PAP).

Analizaron 1174727 citologías cervicales de 938576 mujeres menores de 65 años. La prevalencia de displasia fue mayor en las mujeres menores de 30 años y en las que no tenían historia previa de PAP.

\section{Resultados}

La prevalencia de CCU disminuye a medida que aumenta el número de PAP negativos previos en cualquier grupo etario. De las 32230 mujeres con tres o más pruebas negativas, la displasia de alto grado fue rara en todos los grupos: nueve pacientes con CIN II (0,028\%) y siete con CIN III $(0,22 \%)$. No se reportaron casos de cáncer. Hipotetizando una cohorte de 100000 mujeres y aplicando el modelo matemático de Markov en mujeres con tres o más PAP negativos, el exceso de incidencia de cáncer comparando la estrategia de rastreo anual con la de uno cada tres años se muestra en la tabla 1.
Tabla 1

\begin{tabular}{|c|c|c|c|c|c|}
\hline \multirow[t]{3}{*}{ Edad en años } & \multirow{3}{*}{$\begin{array}{l}\text { Frecuencia } \\
\text { del } \\
\text { papanicolaou }\end{array}$} & \multicolumn{4}{|c|}{$\begin{array}{l}\text { Nuevos casos de cáncer esperados (promedio) luego de } \\
\text { tres PAP previos con resultado normal }\end{array}$} \\
\hline & & \multicolumn{2}{|c|}{$\begin{array}{l}\text { Progresión de displasia GII } \\
\text { a displasia GIII }\end{array}$} & \multicolumn{2}{|c|}{$\begin{array}{l}\text { Progresión de displasia GI } \\
\text { a displasia GII }\end{array}$} \\
\hline & & $\begin{array}{l}\text { Casos } / 100000 \\
\text { mujeres }\end{array}$ & \begin{tabular}{|l|} 
Diferencia \\
atribuible
\end{tabular} & $\begin{array}{l}\text { Casos/100000 } \\
\text { mujeres }\end{array}$ & \begin{tabular}{|l|} 
Diferencia \\
atribuible
\end{tabular} \\
\hline \multirow[t]{2}{*}{ Menores de 30} & Cada 3 años & 9 & \multirow[t]{2}{*}{5} & 9 & \multirow[t]{2}{*}{5} \\
\hline & Anual & 4 & & 4 & \\
\hline \multirow[t]{2}{*}{30 a 44} & Cada 3 años & 5 & \multirow[t]{2}{*}{3} & 2 & \multirow[t]{2}{*}{1} \\
\hline & Anual & 2 & & 1 & \\
\hline \multirow[t]{2}{*}{45 a 59} & Cada 3 años & 2 & \multirow[t]{2}{*}{1} & 1 & \multirow[t]{2}{*}{1} \\
\hline & Anual & 1 & & 0 & \\
\hline \multirow[t]{2}{*}{60 a 64} & Cada 3 años & 1 & \multirow[t]{2}{*}{0} & 1 & \multirow[t]{2}{*}{1} \\
\hline & Anual & 1 & & 0 & \\
\hline
\end{tabular}

Para hacer un análisis de sensibilidad se duplicó la incidencia de displasia, resultando en este escenario dos casos más de cáncer cada 100000 mujeres en el grupo de 30 a 44 años y uno más entre los 45 y los 64 . En cuanto al número de PAP y colposcopías que habría que realizar para detectar un caso de cáncer cervical invasivo con las diferentes estrategias, como fácilmente se puede intuir, es muchísimo mayor para la estrategia de rastreo anual (ver tabla 2).

Tabla 2

\begin{tabular}{l|l|l}
\hline \multirow{2}{*}{ Edad en años } & \multicolumn{2}{|l}{$\begin{array}{l}\text { Cantidad adicional de estudios necesarios para diagnosticar un } \\
\text { caso más de céncer invasivo que con la estrategia "cada tres años" }\end{array}$} \\
\cline { 2 - 3 } & Papanicolaou & Colposcopia \\
\hline \multirow{2}{*}{ Menores de 30 } & 42621 & 2364 \\
\hline 30 a 44 & 69665 & 3861 \\
\hline 45 a 59 & 209324 & 11502 \\
\hline 60 a 64 & No hubo diferencias de efectividad \\
\hline
\end{tabular}

\section{Limitaciones de este estudio}

No conocemos los factores de riesgo para CCU de la población a la cual pertenecen los PAP estudiados. Los datos que se utilizaron para el análisis de incidencia y progresión de la displasia y a los que se les aplicó el modelo de Markov, se basaron en estudios observacionales de mujeres universitarias estadounidenses que no son representativas de la población general (sesgo de selección). La incidencia de displasia grado II que se tomó en cuenta para el análisis fue un poco menor a la que se reporta en otros trabajos ( $1,3 \%$ vs. 1,6 a 4,3\%). En esta cohorte hipotética se asumió una adherencia al rastreo, seguimiento y tratamiento en un $100 \%$, que en al práctica nunca ocurre.

\section{En conclusión}

Aún conociendo las limitaciones de este estudio (que podría subestimar los riesgos) los resultados de este trabajo avalan la estrategia de extender el intervalo inter PAP a tres años luego de tres resultados consecutivos normales en una población que tiene acceso al sistema de salud y está educada en salud (recordemos que en este trabajo las incidencias fueron tomadas de poblaciones con educación universitaria y que se asumió una adherencia del 100\%).

Cuando el equipo de salud tiene información sobre el nivel socioeconómico y educativo, así como sobre los factores de riesgo de CCU de cada paciente que tiene a su cargo (ej. número de parejas sexuales) y cuando existe adecuada adherencia, no parece demasiado difícil poner en práctica estas conclusiones, evitando la sobreprestación y ahorrando costos innecesarios. Sin embargo, conociendo la poca accesibilidad al sistema, la pobre concientización sobre los cuidados de la salud de la mujer y la incertidumbre de poder sostener la continuidad de la estrategia de rastreo, es más difícil extrapolarlas a la población argentina. Siendo la cobertura nacional con PAP muy deficitaria, todavía nuestro desafío sigue siendo lograr que todas las mujeres accedan al PAP y que el CCU no sea una de las principales causas de mortalidad de la mujer argentina.

\section{Dra. Mariela Barani - Dra. Alejandrina Lo Sasso [ Unidad de Medicina Familiar y Preventiva del Hospital Italiano de Buenos Aires ]}

\section{Referencias}

1. Screening for Cervical Cancer: Recommendations and Rationale, US Preventive Services Task Force, April 15, 2003.

2. Programmatic Guidelines for Screening for Cancer of the Cervix in Canada, 1998.

3. Recommendations on Cancer Screening in the European, Advisory Committee on Cancer Prevention, Conference on Screening and Early detection of Cancer, Vienna 18 th -19 th November 1999 